\title{
Neuroanatomical Aspects of the Temporo-Parieto- Occipital Junction and New Surgical Strategy to Preserve the Associated Tracts in Junctional Lesion Surgery: Fiber Separation Technique
}

\author{
Huseyin BICEROGLU1', Ali KARADAG² \\ ${ }^{1}$ Ege University, Faculty of Medicine, Department of Neurosurgery, Izmir, Turkey \\ ${ }^{2}$ Menemen State Hospital, Department of Neurosurgery, Izmir, Turkey \\ Corresponding author: Huseyin BICEROGLU huseyin.biceroglu@gmail.com
}

\section{ABSTRACT}

AIM: To describe the location of each white matter pathways around the temporo-parieto-occipital junction (TPOJ) and the dissections performed using focal fiber dissection and fiber separation techniques (FST). These areas play an important role in human language processing and high-level brain functions. Thus, it is important to identify the association of the fascicles as well as their courses to plan safe and effective surgical target vectors.

MATERIAL and METHODS: Thirty formalin-fixed human hemispheres and two formalin-fixed human heads were dissected with focal fiber dissection and FST under 6-40x magnification using a surgical microscope and real-time magnetic resonance imaging navigation system. Two- and three-dimensional anatomical and surgical pictures were obtained and processed using high dynamic range photography (Photomatix) and were correlated to radiological images of the case with glioblastoma of the TPOJ.

RESULTS: The trajectory and connectivity of these fibers as well as their surgical importance in performing FST in cadaver dissections were demonstrated with correlation of the surgical, radiological, and anatomical images.

CONCLUSION: The microanatomy of the TPOJ and related structures, emphasizing the position of each white matter pathway, has been described. Understanding the composition of each region is critical in preventing intraoperative brain injury that could lead to functional deficits.

KEYWORDS: Brain, Temporo-parieto-occipital junction, White matter, Fiber separation technique, Tractography, Neuroanatomy

ABBREVIATIONS: AG: Angular gyrus, AF: Arcuate fasciculus, ASA: Anterior cerebral artery, DTI: Diffusion tensor imaging, EC: Extreme capsule, IC: Internal capsule, FST: Fiber separation technique, IPL: Inferior parietal lobule, IFOF: Inferior frontooccipital fasciculus, ILF: Inferior longitudinal fasciculus, MRI: Magnetic resonance imaging, MCA: Middle cerebral artery, MdLF: Middle longitudinal fasciculus, SLF: Superior longitudinal fasciculus, SMG: Superior marginal gyrus, STG: Superior temporal gyrus, SD: Standard deviation, TPOJ: Temporo-parieto-occipital junction, WHO: World Health Organization.

\section{INTRODUCTION}

$\mathrm{T}$ The temporo-parieto-occipital junction (TPOJ) is a highly complex area, which is associated with language, sensory, motor, and visual fascicles of the brain, and is the core where the temporo-parieto-occipital lobes meet $(11,41)$. Better understanding of the anatomy of the important functional brain structures is critical in surgical planning $(39,41)$. Crucial eloquent areas are located around the TPOJ and can be identified by diffusion tensor imaging (DTI), and 
the areas to preserve during the surgery include the sensory, motor, visual, and language areas $(39,52)$. Obtaining total tumor excision borders, progression-free survival after total resection, and overall survival indicate the potential clinical importance of the novel fiber dissection techniques and DTI images $(39,41,52)$. The TPOJ controls several functions of the brain, including language, visual-spatial recognition, writing, reading, symbol processing, calculation, self-processing, working memory, musical memory, and face and object recognition $(11,53)$. Anatomical knowledge must be excellent to perform successful surgery to the TPOJ and preserve this region $(11,13)$. Former studies have emphasized the traditional "eloquent" areas (Wernicke's and Geschwind's territories), but recent researchers have demonstrated that the TPOJ has many widely variable connections beyond the known anatomical landmarks and variability $(7,12,13,44)$. Thus, this hypothesis has evidences of further remodeling of the surrounding structures and provides ideal neuro-functional compensation after pathological events, including ischemic accidents or tumor infiltration $(11,12,51)$. The relationship between the TPOJ and Wernicke's area, angular gyrus (AG), central sulcus, Heschl's gyrus, superior and inferior limiting sulcus, superior longitudinal fasciculus (SLF), arcuate fasciculus (AF), middle longitudinal fasciculus (MdLF), inferior longitudinal fasciculus (ILF) and inferior fronto-occipital fasciculus (IFOF), internal capsule (IC), and extreme capsule (ExtC) have been described by many researchers based on performed fiber tract dissections and DTI experiments $(11,15,31,37,53)$. SLF is one of the most complicated pathways of the brain $(30,37)$. AF connects the frontal with the temporal cortex. There are three main SLF components (SLF I, II, III) that connects the frontal lobe with parietal lobe $(5,7,16)$. The SLF II provides a connection between the dorsolateral prefrontal and premotor cortex with the AG; the SLF III also connects the ventrolateral frontal cortex with the SMG. The MdLF connects the inferior parietal lobule (IPL) with the temporal cortex, thereby being distinct from the posterior portion of the $\operatorname{AF}(5,16,24,37)$.

In this study, the courses of each white matter pathways around the TPOJ were described, paying special attention to their relationship to the areas that are responsible for language functions. Fiber separation technique (FST) can be applied during surgery in patient with glioblastoma of TPOJ, and this identified region may be used as a landmark to estimate the position of each set of fibers. Nevertheless, relative to the well-known directions of the large fascicles, little data exist pertaining to the fibers associated with the TPOJ. The importance and effect of this anatomy and FST in planning surgical strategies applicable to patients with glioblastoma in the TPOJ of the dominant hemisphere are discussed.

\section{MATERIAL and METHODS}

\section{Anatomical Stage}

The anatomical dissections were performed at the McKnight Brain Institute, Department of Neurosurgery, Florida, USA. Thirty formalin-fixed human hemispheres and two formalinfixed human heads were dissected. The traditional fiber dissection technique was first described by Klingler. Based on this technique, all specimens were frozen at $-16^{\circ} \mathrm{C}$ for at least 15 days and were allowed to thaw under water for several hours. Colored latex was injected in the vessels of the brain and fiber dissection is performed after freezing (27). Controversially, in our study, freeze-in water technique, in which all specimens were soaked in water at for least 4 days and allowed to freeze in the water for 3 months, was used. FST (a modified technique of Klingler's), which enabled us to separate individual fibers without destroying them, was performed. After completing the freezing process, the specimens were defrosted, and the fiber tracts were dissected and separated using micro dissectors under 6x40x magnifications through a Zeiss surgical microscope (Carl Zeiss AG, Germany). The specimens were preserved in alcohol solution during the dissection sessions. The hemispheres were dissected in a stepwise manner, from lateral to medial and anterior to posterior, and, at the same time, in the same specimen using the fiber dissection and separation techniques and the microscope until the TPOJ and related fibers were revealed. Three-dimensional photography (Canon T5 Rebel digital camera, Canon, Tokyo, Japan) and the Stealth Station ${ }^{\circledR}$ surgical navigation (Medtronic) system were used; two- and three-dimensional anatomical and surgical pictures were obtained and processed using HDR photography program $\left(\right.$ Photomatix $\left.{ }^{\circledR}\right)$. Cadaveric magnetic resonance imaging (MRI) provided real-time images to document the fibers and assess their important during the dissection. The position of the fiber tracts found in each deeper step of these dissections was noted according to their depth from the overlying cortical gyri. The topographic location and the subcortical fiber tracts in relation to the overlying gyrus or gyri, especially around the TPOJ, were discussed.

\section{Radiological Stage}

The World Health Organization (WHO) grade IV glioblastoma of the TPOJ was studied using brain MRI and DTI (a singleshot multislice spin echo-echo planar sequence with the following attributes-diffusion sensitization, $1300 \mathrm{~s} / \mathrm{mm}^{2}$; repetition time, 9577 milliseconds; voxel size, $2 \mathrm{~mm}^{3}$ ). The DTI data sets and anatomical MRI were analyzed with the use of fiber track software for diffusion tensor analysis and fiber tracking from MR Work Space (Philips Healthcare). To analyze the fiber spatial relationship in the TPOJ, the fibers were reconstructed and correlated to real-time MRI navigation. Each fiber system of peripheral region was dissected using MRI navigation that enabled the researchers to identify the surgical views, neighborhood relationships, and depths of the dissected fibers. MRI of two heads with intact skull base is obtained through the navigation system out of the laboratory and the radiological images are transferred to the navigation storage. The selected standard landmarks (orbital rim, lateral epicanthus, and nasion) are defined. The device is confirmed analytically and anatomically and then dissection is performed.

\section{Case Description}

A 50-year-old, right-handed man was admitted due to persistent headache with moderate working memory difficulties and normal language functions. The patient was cooperative and oriented to time, place, and person. 
Upon presentation, a 4/5 right hemiparesis was observed. Preoperative $\mathrm{MRI}$ with contrast revealed a glioblastoma extending from within the superior gyrus of the temporal lobe and infiltrated to the parietal lobe and antero-superior portion of the occipital lobe (Figure 1A-C). DTI tractography showed that the tumor directly infiltrated the TPOJ, with contact to SLF I and III, AF, EC, IFOF, and IC (Figure 1D-F). Tumor operation with total resection was performed through a transcortical approach. Through the application of FST, the IC and AF fibers were separated from the tumor using a blunt suction tube and blunt periosteal elevator medially and anteriorly, respectively. FST is based on a principle that a vectoral force parallel to the selected fiber system can separate the tumor at the tumorfiber border zone without destroying the fibers because the strength of the tumor is occasionally different from the fiber system (Figure 2A, B). The WHO grade IV glioblastoma (IDH mutant, ATRX negative, OLIG2 positive) was detected during the final pathological investigation. The patient was discharged with mild postoperative dysphagia but completely recovered one month after surgery. No other postoperative deficits were detected. Axial T1-weighted contrast enhanced (Figure 1G-I), and T2-weighted MRI (Figure 1J) showed the total excision of the tumor with changes due to radiotherapy at the second year after surgical intervention.

\section{RESULTS}

The TPOJ can be described as the intersection point of the three lobes and is related to eloquent brain areas that are responsible structures for important functions. Brain
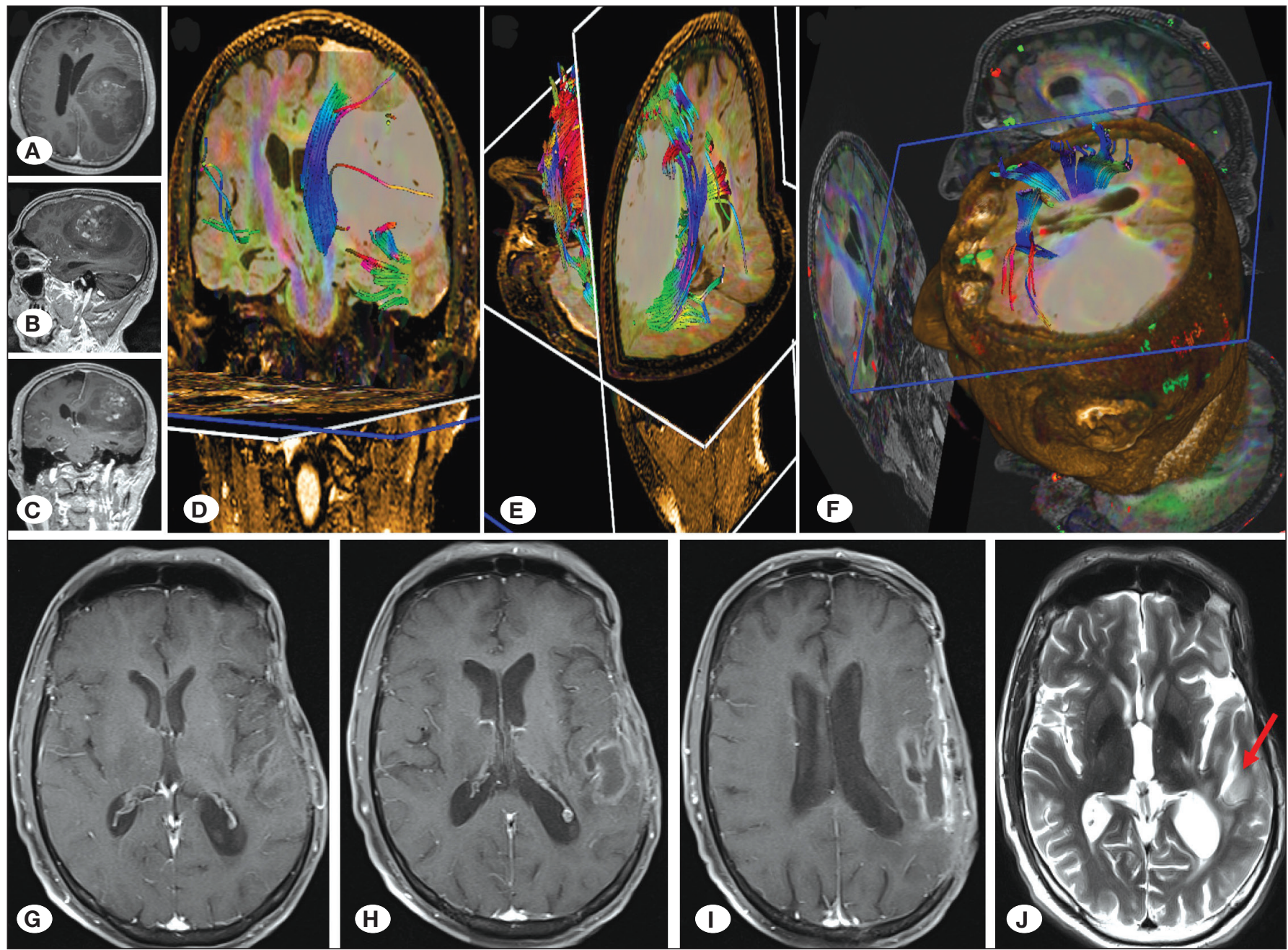

Figure 1: Pre-operative axial (A), sagittal (B) and coronal (C) T1-weighted with contrast MRI visualized a left glioblastoma multiforme that is located on the TPOJ and surrounded by marked edema and infiltrative tumor, which causes significant mass effect. The lesion demonstrates aggressive imaging features, including signal heterogeneity, necrotic center, and peripheral enhancement. The TPOJ is viewed from a posterior lateral and superior perspective, demonstrating the complex fiber tracts organization of this area using the DTI reconstruction. Fibers of the corresponding fasciculi are shown. Pre-operative DTI showed the relationship between the tumor and the surrounded fibers, especially demonstrated the covering the IC fibers medially (D, E, F). At 2 years, post-operative axial T1-weighted with contrast MRI (G, H, I) showed the subtotal resection and the radiation necrosis in the TPOJ area. Axial T2-weighted MRI revealed postoperative changes at the surgical cavity and the source the tumor located very closed to Wernicke's Area (J) (arrow). 
structures that are associated with the TPOJ and surrounding fibers were preserved during the dissection to analyze the courses and elongations of the tracts between the superficial and deep cortex that are important in language functions as well as between the tracts. We exposed the so-called $U$ fibers connecting the gyri to another under their respective sulcus, which lay under the white matter, after removing the entire cortex. Parallel, closed-packed fibers around the TPOJ have a long structure and, the pathways of those remain discrete as they course through their long and wavy structure toward the area being targeted. In addition, the fibers that are associated with the posterior portion of the SLF from the AF can be isolated. At the TPOJ, a clear plane of cleavage was identified between the fibers of the AF, which have a superior-inferior orientation, and the related fibers of the SLF II, SLF III, IC, and ExtC (Figure 3A), and the FST revealed the deep IC fibers (Figure 3B-D). Superficial long connective fascicles running through the TPOJ is known as SLF. The SLF is formed by three compartments and is the first superficial pathway that revealed below the Ufibers (Figure 4). The SLF I has a relationship with the association fiber tracts and provides a connection between the superior horizontal fibers and superior parietal lobe (Figure 5). Fiber dissection from the lateral surface to the medialdeep structures of the left hemisphere reveals that the three components of the SLF (I, II, II) are located under the superior, middle, and inferior frontal gyri, respectively (Figures 4-6, 9A, B). $A F$ has two parts-superficial and deep. They are located deeper than the posterior segment of the SLF and connect the posterior temporal lobe with the frontal lobe (Figure 8A, $B)$. The perisylvian cortex of the frontal, parietal, and temporal lobes have a close relationship with the AF that is a lateral associative bundle composed of long and short fibers. ILF is the other associated fascicle with the TPOJ region that is horizontally oriented, runs along the inferior temporal and occipital lobes, and serves as a connective pathway between them. IFOF is located at the antero-inferior direction and runs within the frontal lobe, insula, temporal stem, and sagittal stratum; it also connects the frontal and occipital lobes. If dissection continues to the TPOJ, IFOF can be separated from the uncinate fascicle fibers, which is an association system connecting the frontal lobe with medial and anterior temporal lobe. Removing the white matter of SLF and then following the SFOF fibers demonstrated the relationship of the deeper corona radiata and $I C$ fibers. Structure of IC is formed by IC fibers, which originate from different parts of the cerebral cortex, and is situated medial to the lentiform nucleus and lateral to the caudate nucleus (CN) and thalamus. Additionally, $\mathrm{CN}$ lies in the lateral periventricular area. This area is on the main trajectory of the IC fibers. It involves the associative region that sits lateral to the $\mathrm{CN}$ above the upper border of the lentiform nucleus. The main characteristic of the ExtC is having a long association fiber pathway of white matter; it also has communication to the claustrum, insular cortex, inferior frontal gyrus, and middle-posterior portion of the STG and forms a bridge between them (Figure 7). MdLF was clearly differentiated from the AF because it is deeply located and has an anteroposterior and superior orientation, connecting the superior temporal gyrus with the parietal lobe.

\section{DISCUSSION}

The cerebral tissue has different characteristics and can be described as remarkably delicate and intricate $(22,25)$. Therefore, most of the time, the main aim of the neurosurgeons is to preserve "nature's masterpiece, the human brain" using accurate and safe techniques $(22,25)$. This is the first study that utilizes three-dimensional HDR for documentation and 3 Tesla MRI navigation in performing focal fiber dissection and focal FST of the human head cadavers with the application of the novel freeze-in water technique. The TPOJ and FST with surgical case correlation have been performed to identify which arteries and eloquent brain areas are preserved. Little
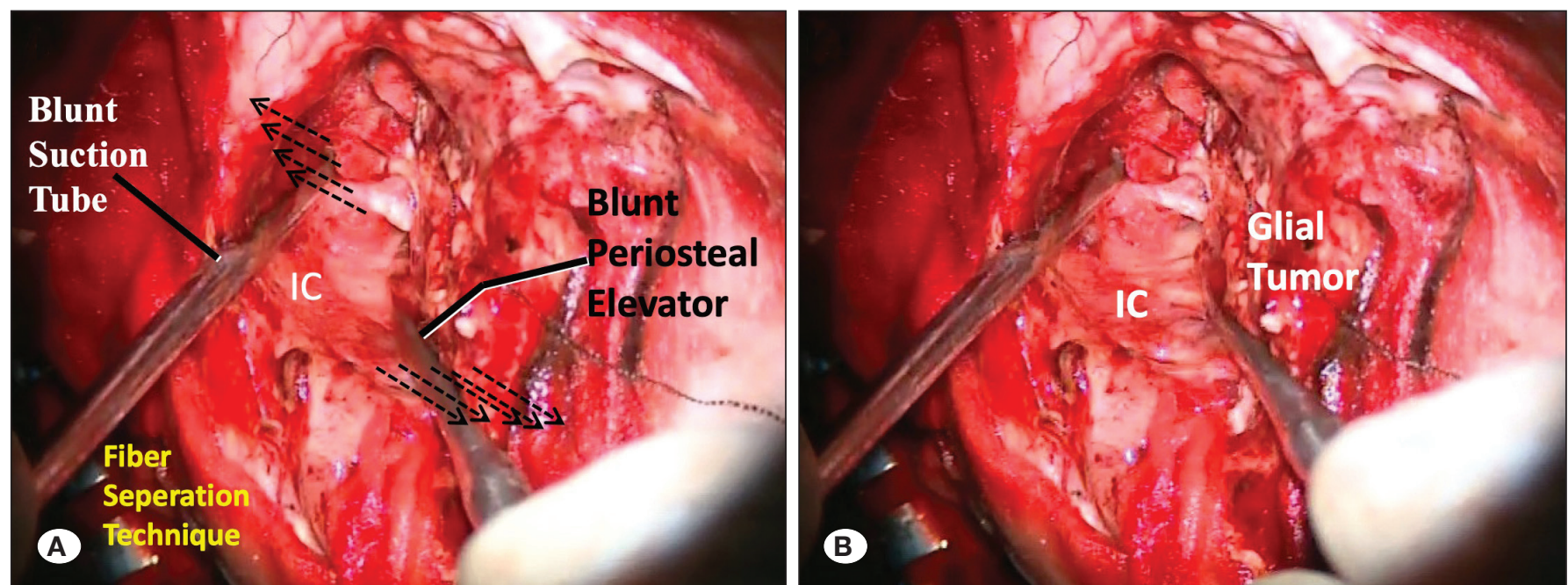

Figure 2: Fiber Seperation Technique is based on a principle that an vectoral force parallel to the selected fiber system can separate the tumor at the tumor-fiber border without destroying the fibers because the strength of the tumor is occationally different than the fiber system. FST can be seen in the intra-operative images. The IC fibers were separated step-by-step from the tumor without destruction using this technique (A, B). 
is known concerning the relations, courses, and connections of the fibers of the white matter; the fiber systems are difficult to follow, and descriptions are largely based on anatomical dissections and radiological studies of fiber tracts. Most of what is known today about the pathways that connect neurons to different brain regions has been discovered using neuroanatomical tract-tracing techniques. In this present study, fiber tract dissection was performed to reveal the fiber courses of the fibers and anatomical connectivity of the TPOJ. Additionally, an illustrative case with glioblastoma using its surgical pictures, DTI, and brain MRI images was presented to support the importance of the fiber tract dissection without coagulation of the cerebral tissue. The significance of the anatomical knowledge in decreasing the brain damage was discussed. Understanding the topographic locations and trajectories of the associated fibers with the TPOJ is essential for successful surgical access and planning a surgical strategy in patients with tumors and vascular lesions. Many research studies about the white matter of the brain have focused on microstructural niceties $(6,23)$. Conversely, we emphasized the characteristic and nuances of medium and large fiber tracts of the TPOJ and surrounding fascicles as well as their functional and anatomical relationship to expose the surgical effects. We have described the white matter connections with
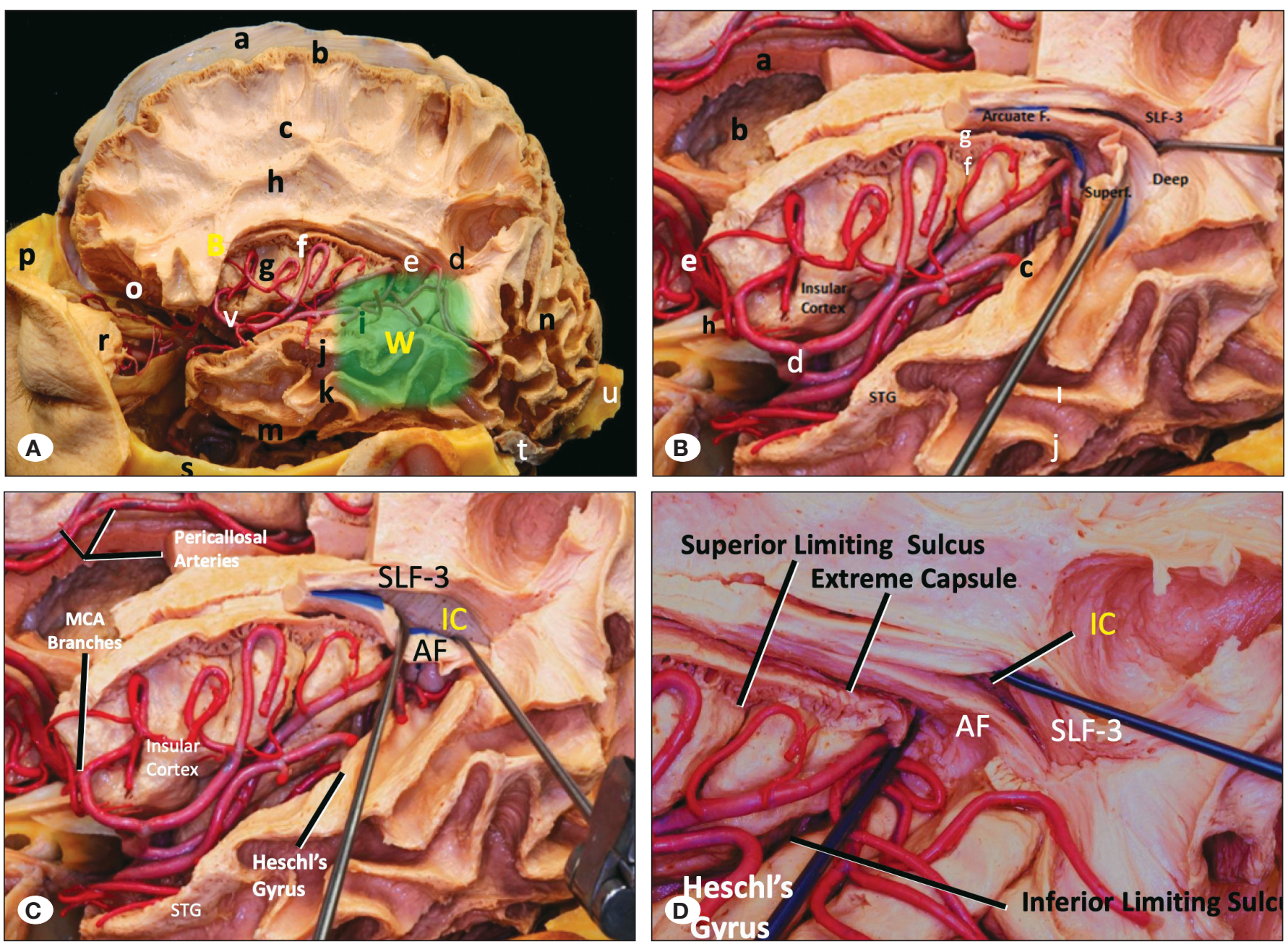

Figure 3: A) The initial stage of dissection: Removing the cortical white matter and the arachnoid mater to expose the indirect and direct components (Wernicke's area, angular gyrus, central sulcus, Heschl's gyrus, superior and inferior limitan sulcus, SLFs, AF, MdLF, ILF, IFOF, IC and EC) of TPOJ during a dissection of a left hemisphere. Red zone indicates the Broca's area and Green Zone the Wernicke's Area.a. falx cerebri, b. cortex, c. SLF-1 fibers, d. SLF-3 fibers, e. Arcuate Fasciculus, f. Superior Limiting Sulcus, g. Insular Cortex, h. SLF-2 fibers, i. Heschl's Gyrus, j. Decorticated Superior Temporal Gyrus, k. Decorticated Middle Temporal Gyrus m. Decorticated Inferior Temporal Gyrus n. Decorticated Occipital Lobe o. Orbitofrontal Cortex p. Frontal Sinus r. Eye , s. Zygomatic Arc, t.Transvers Sinus, u. Occipital Protuberans B) AF lies just underneath the SLF-3 and latera to Internal Capsule. AF has two separate components: Superficial and deep parts, a. Corpus Callosum, b. Caudate Nucleus, c. Heschl's Gyrus, d. Middle Cerebral Artery, e. Anterior Cerebral Artery, f. Superior Limiting Sulcus, g. Extreme Capsule, h. Olfactory Nerve. C) Arcuate Fascicule's Fibers are retracted with fiber seperation Tecnique to expose Internal Capsule Fibers. Internal capsule is located medial to Superior Longitudinal Fasicule 3 and Arcuate Fascicule (D). Arcuate Fascicule is located just above the jucton of Superior Limiting Sulcus and İnferior Limiting Sulcus. SLF-3 fibers and Arcuate Fascicule Fibers are seperated and SLF-3 fibers are elevated upwards in order to expose Internal Capsule. 


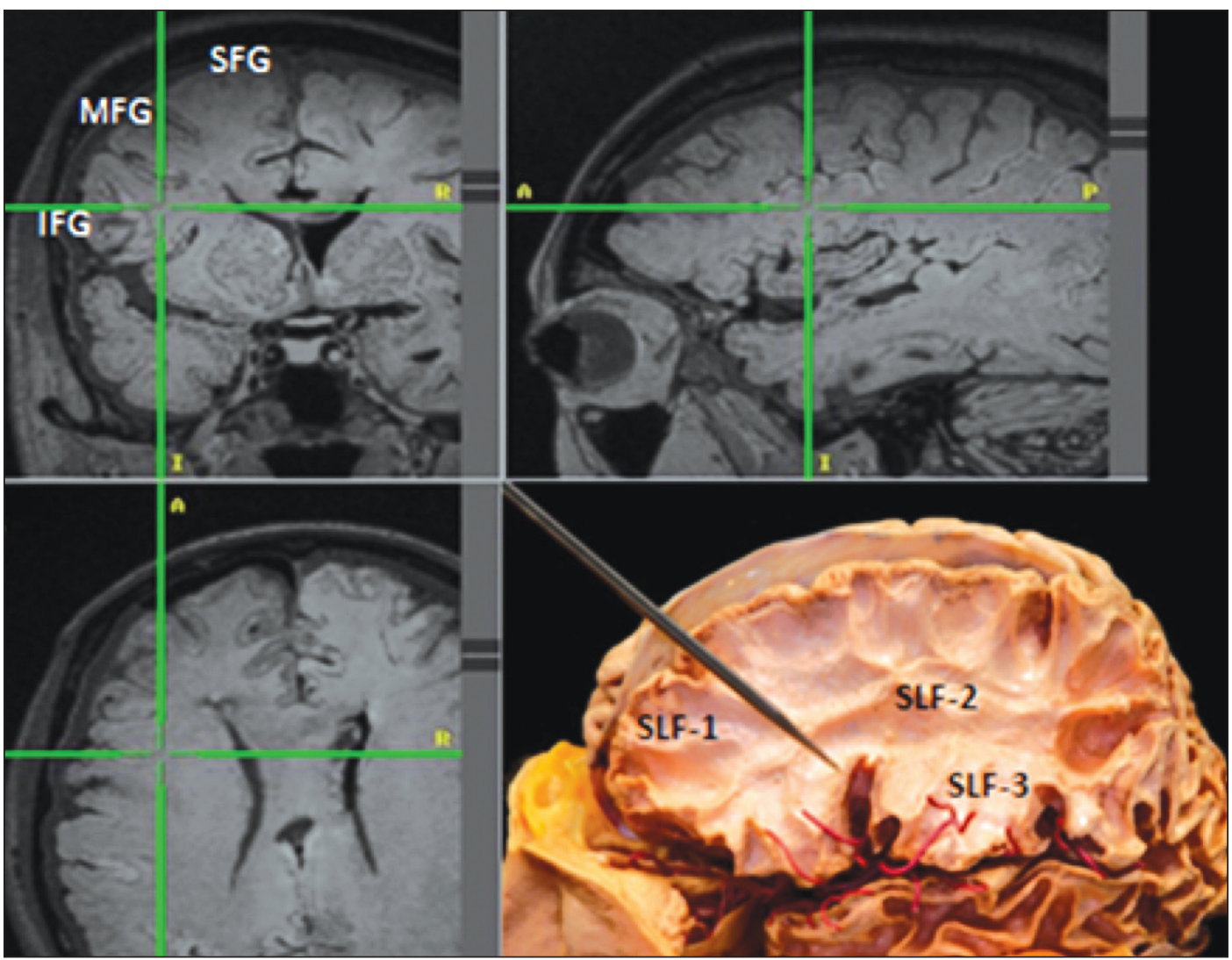

Figure 4: MR Navigated dissection demonstrated the location of SLF-3 fibers: SLF-1 is located under Superior Frontal Gyrus, SLF-2 is under Middle Frontal Gyrus and SLF-3 is located under Inferior Frontal Gyrus.

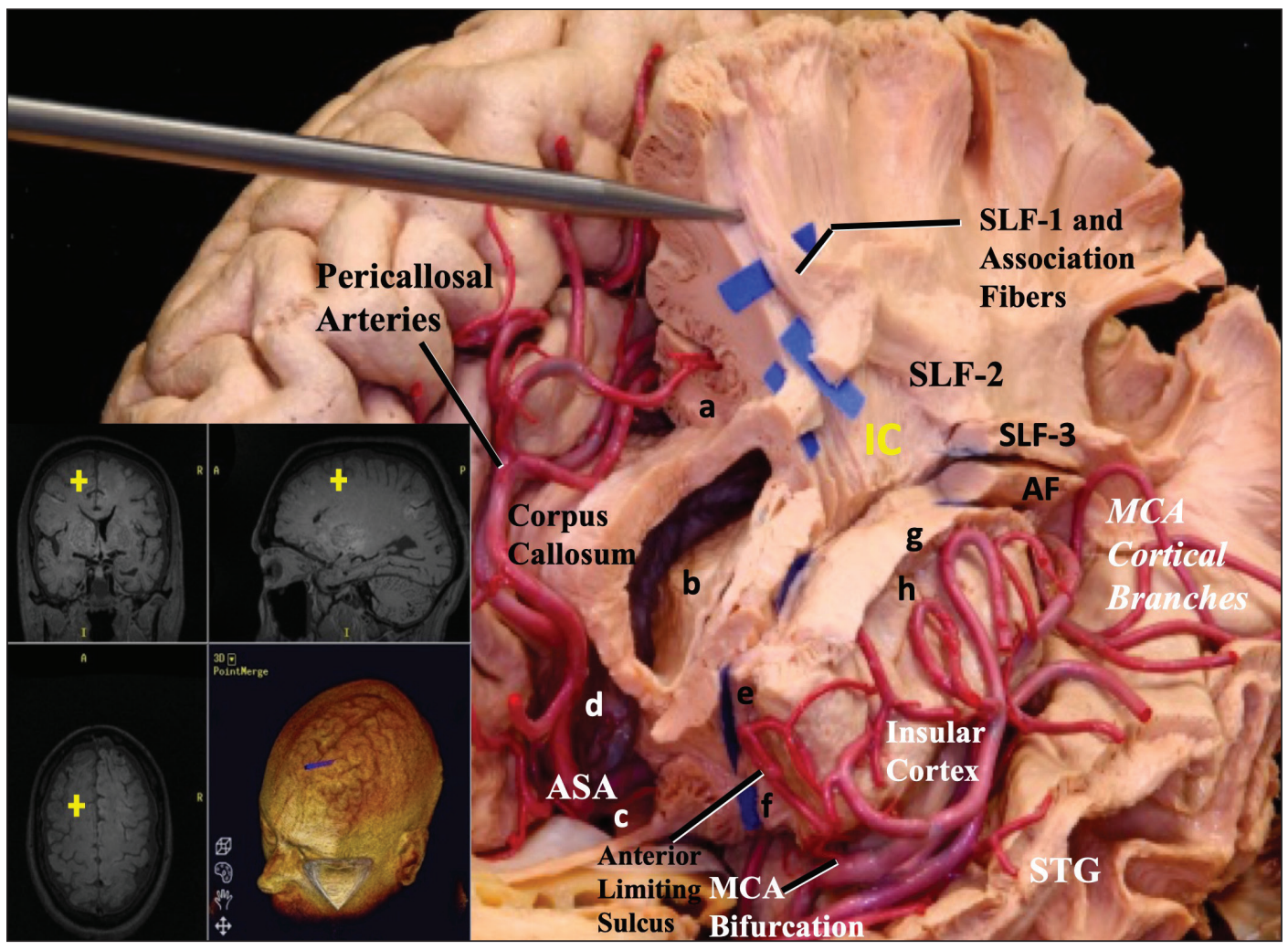

Figure 5: SLF-1 and association fibers were exposed using MR Navigation, lateral view. SLF-1 in the medial section of the hemisphere. It is the main connection between the superior parietal lobule and anterior cingulate cortex and is associated with upper part of the cingulate sulcus above the cingulum. Navigation probe indicates the superior longitudinal fasciculus 1 which is located under superior frontal gyrus. a. Cingulum Bundle, b. Caudate nucleus, c. Optic Nerve, d. Diagonal Band of Broca e. İnerior Frontooccipital Fasciculus f. Uncinate fasciculus g. Extreme Capsule h. Superior Limiting Sulcus

ASA: Anterior cerebral artery. MCA: Middle cerebral artery.

STG: Superior temporal gyrus. IC: Internal capsule. 
respect to major underlying pathways using DTI and FST $(26,53)$. In addition, the TPOJ was investigated, and the data were analyzed by evaluating the surgical process performed to lesions of this complex area (26). MRI and functional works are essential and sufficient tools necessary to reveal the relationship between the lesion and fascicles. There are many variable components that cause brain damage during the surgery; therefore, correlation of the dissections and the radiological data may help increase the surgical orientation. Moreover, solid tumors, including high-grade astrocytoma or glioblastoma, show more aggressive features, including heterogeneous parenchymal necrosis, surrounding edema,

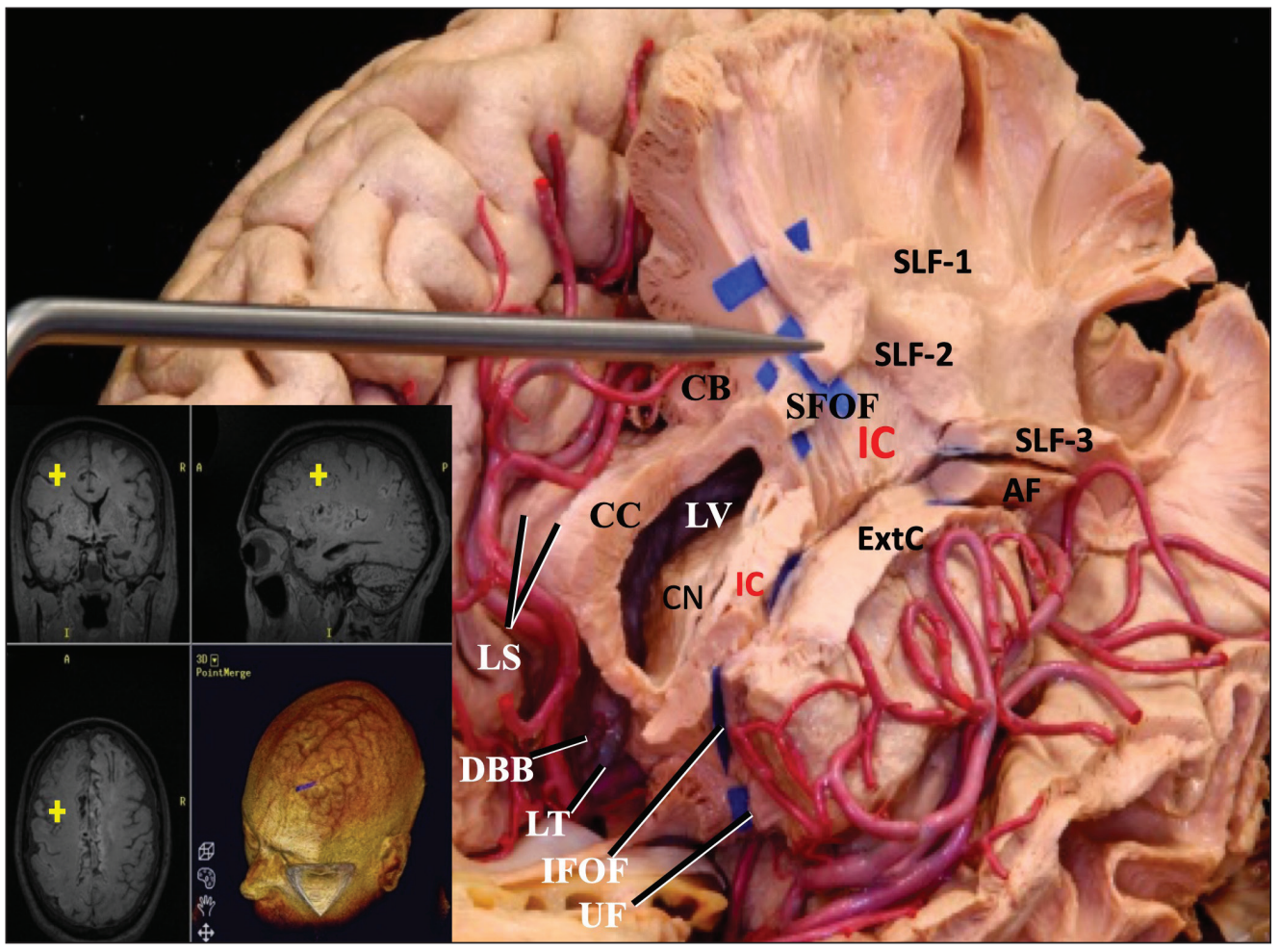

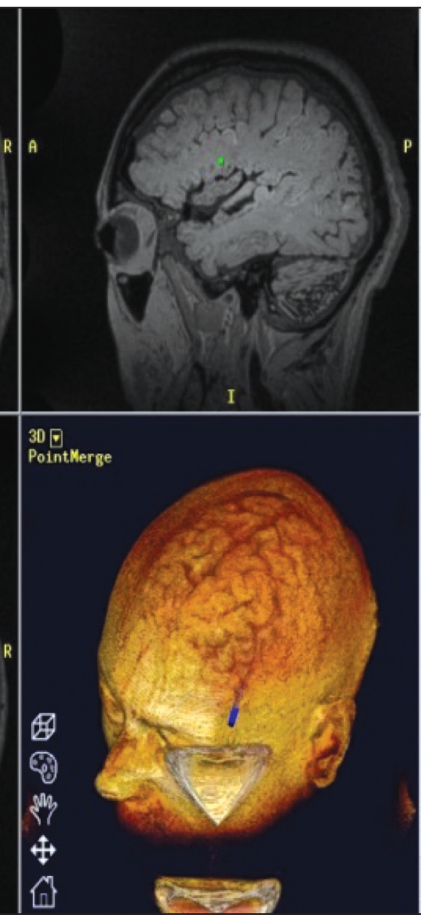

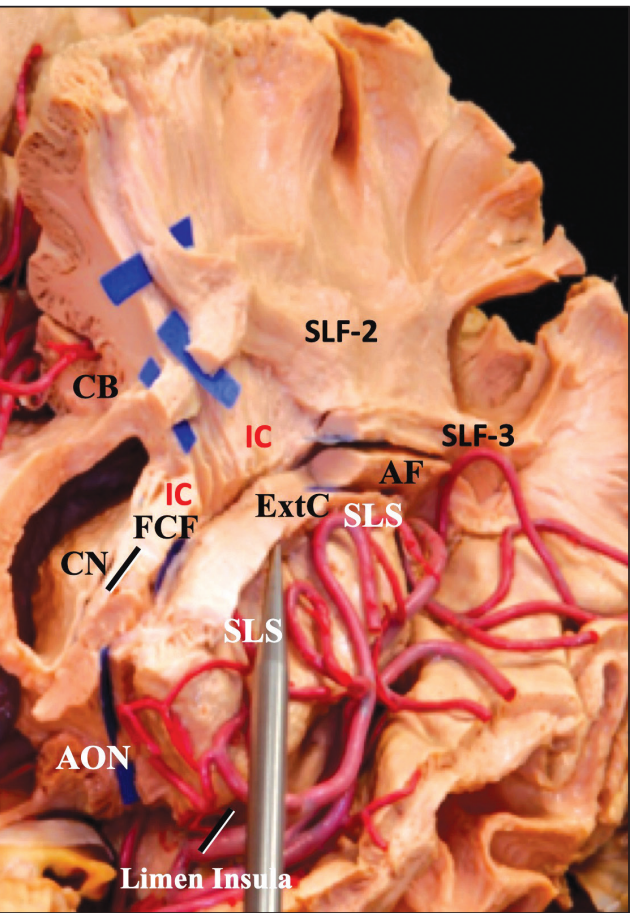

Figure 7: Extreme Capsule is an association fiber system between Insular Cortex and other cortical areas. Extreme capsule is located just above the superior limiting sulcus. Navigation probe is located just above the superior limiting sulcus and indicates the association fibers of extreme capsule. $\mathbf{C N}$ : Caudate nucleus. FCF: Frontocaudate fibers. IC: Internal capsule fibers. SLS: Superior limiting sulcus. AON: Anterior olfactory nucleus. CB: Cingulum bundle. 

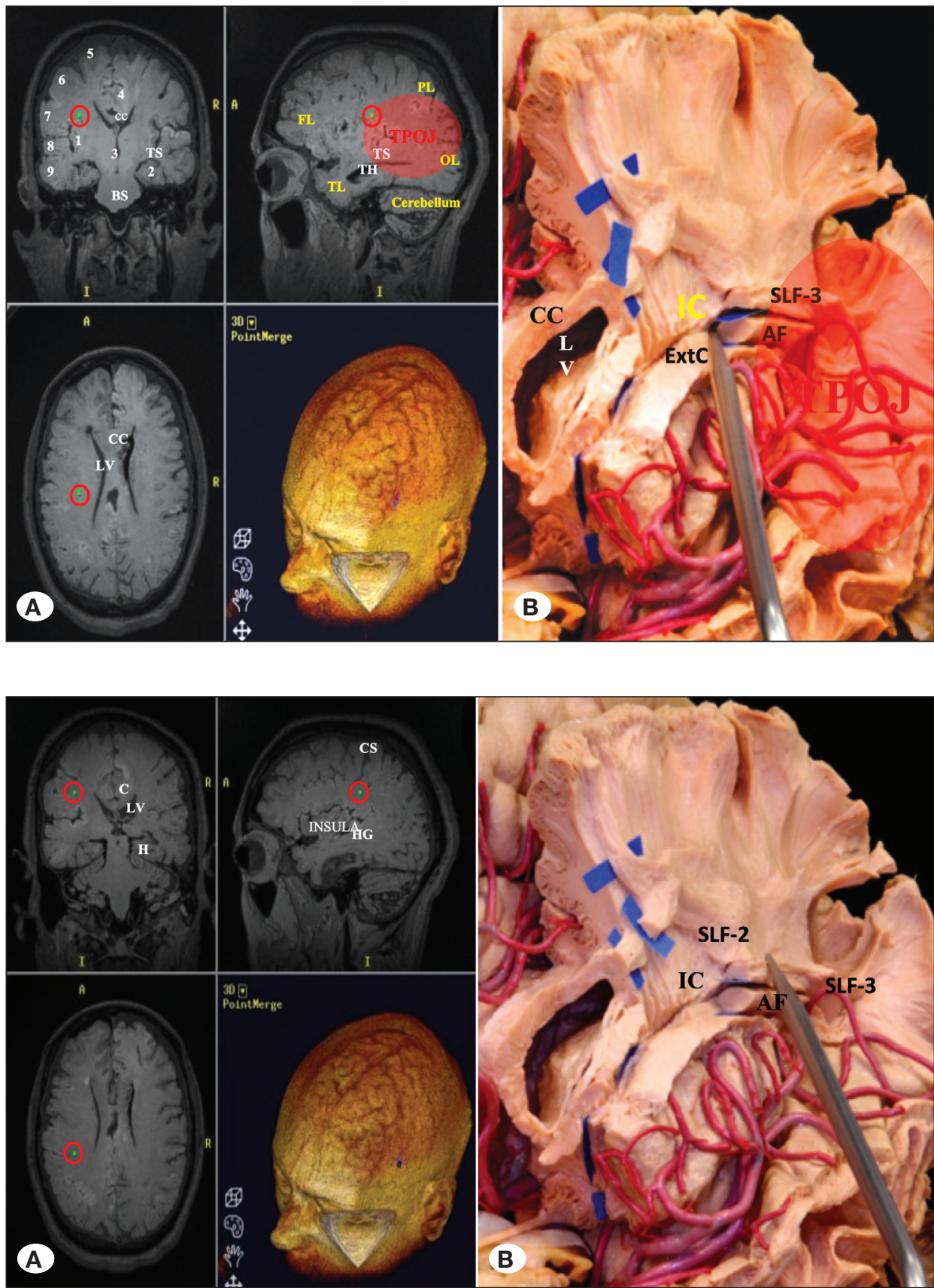

Figure 8: MR navigated

demonstrated the junction of the AF and IC fibers. The AF, is a complex white matter bundle that contains contributions that contains lateral temporal cortex with frontal cortex that arches around the Sylvian fissure. 1. Insular cortex 2. hippocampus 3. Third ventricule 4. Cingulate Gyrus 5. Superior frontal gyrus 6 . middle frontal gyrus 7. inferior frontal gyrus 8. superior temporal gyrus 9. middle temporal gyrus BS: Brainstem. TS: Temporal stem. CC: Corpus callosum. FL: Frontal lobe.

PL: Parietal lobe. OL: Occipital lobe. TL: Temporal lobe. TH: Temporal horn. TPOJ: Temporo-parietooccipital juctional area. LV: Lateral ventricule.
Figure 9: Navigation probe is located between the SLF-2 and SLF-3 Fibers just above the arcuate fasicule and lateral to internal capsule.

HG: Heschl's Gyrus. CS: Central sulcus. H: Hippocampus. infiltrative margins, significant mass effect, and marked enhancement (9,26). Glioblastomas show invasive and malignant characteristics. FST follows the fibers of the white matter pathways and allows isolation of individual fiber bundle from the tumor. Therefore, in general, subtotal resection is possible, and combined treatment that includes surgery along with chemoradiotherapy is preferred. MRI with contrast helps surgeons identifying the visible part of these types of tumors. DTI tractography can decrease damage of the eloquent brain areas. In this case, awake craniotomy can be considered as an alternative technique as it can minimize the risk of complications $(4,18,20,46,48)$. But minor intra-, post-operative complications in awake brain surgery should be considered as a disadvantage $(18,20)$. The large tumor was dissected from the IC fibers and FST, which is based on a principle that a vectoral force parallel to the selected fiber system can separate the tumor at the tumor-fiber border zone without destroying the fibers because the strength of the tumor is occasionally different from the fiber system, was used parallel to the IC fibers. AF fibers were also preserved using FST so that the undamaged brain tissue is forced to be separated from pathological tissue using the blunt periosteal elevator because the white matter has different resistances from tumor involved. Based on our experience, awake craniotomy 
should not be considered because the tumor was too large and DTI tractography demonstrated involvement of the IC fibers that run medial of the tumor (Figure 1D-F). Since the tumor was very large, it pushed and changed many eloquent areas and decreased the surgical orientation; this prevented us from seeing the tumor cleavage for us to be able to perform subtotal resection even with the use of DTI. The TPOJ has many complex connections associated with AF, SLF III, and IC in a small area. DTI tractography should be obtained and then awake surgery can be performed to minimize brain damage, and when the brain tissue demonstrated maximum shift, FST can also be used. Our experiences showed that the dissection technique described by Klingler destroyed the fibers more than FST. It is critical to minimize the brain damage when the awake craniotomy and DTI could not be of any help. Tractography displayed the meeting point of the temporo-parieto-occipital lobes and the connections among the occipital, temporal and parietal lobes. Fiber dissection was started at the lateral hemispheric surface, and major anatomical landmarks were identified before splitting the brain. Our dissections indicate that the posterior part of SLF II and III connects to the posterior temporal lobe and the inferior parietal lobe and is related to AF. The functions of these fibers are critical in wide frontoparieto-temporal connections $(11,37,53)$. SLF I is located between the medial posterior parietal region-superior parietal region and the supplementary motor area of the frontal lobe, dorsal area 9, and dorsal area $6(30,40)$. The SLF II lays lateral of the corona radiata and superior of the insula and remains at the posterior craniocaudal of the AF. The AG is connected to the temporal lobe by the MdLF and is also the point of origin of the SLF II. SLF II fiber tract courses in the anteroposterior direction with the horizontal portion of the AF, passes through the pre and post central gyrus, and ends in the dorsolateral prefrontal cortex. In addition, SLF III courses through the SMG and has a close relationship with these fibers $(24,39)$.

SLF I plays a critical role in regulating high motor functions, transporting somatosensory and kinesthetic information, and initiating motor activity. Working memory, semantic and phonologic processing, number manipulation, response inhibition, automatically capture attention, decision making, and emotion processing functions have been found to be associated with SLF I $(30,40,43,45)$. SLF II is responsible for visual-spatial attention and awareness task-oculomotor functions in both hemispheres. Damage to the SLF II can affect the spatial working memory and cause attention problems, and this is can be manifested as Neglect and Gerstmann Syndrome $(10,30,49,50)$. SLF III is associated with saccades, volumetric-oriented attention, mental imagery, and motor sequence functions. It is responsible for lingual articulation on the dominant hemisphere and is associated with visual-spatial attention, prosody, and musical memory in the nondominant hemisphere. Damage to this pathway can cause spatial hemineglect $(29,30,50)$. The Wernicke's area is defined as the area responsible for understanding words and sentences, and the Broca's area is responsible for processing grammatical structures to be transformed into speech content and understanding complex sentences. According to the Wernicke-Geschwind model, these areas have been found to be associated with AF (8). AF plays a key role in understanding sentences with have complex grammar and in analyzing syntax processes $(19,33)$. Moreover, ExtC has fibers that lie medially to the insula and laterally to the claustrum and is located in the area between the inferior frontal region and the STG, which is extended to the IPL $(14,24,31)$. ExtC is the main pathway between the Wernicke's temporal language and ventrolateral frontal area (42). ExtC and MdLF are additional ventral pathways. However, many DTI tractography studies cannot differentiate ExtC from IFOF. The role of these fibers in language functions is not clear yet $(31,42)$. The inferotemporal part of ILF is related to object identification, and the parahippocampal gyrus part is related to correlation of sensory inputs in object- and regionrelated memory $(35,38)$. ILF fibers that are located under the superior temporal sulcus contribute in analyzing visual movements $(17,28)$. ILF associated with the parietal lobe is helpful in facilitating visual-spatial and motion analysis (47). ILF also links the occipitotemporal regions to the early stages of the visual processing flow. Feedback connections from the inferotemporal cortex to the occipital lobe and amygdala play a role in attention, memory, and visual matching $(2,3)$. IFOF extends in the same direction as MdLF in the temporal lobe. As MdLF enters the temporal opercular space, by partially leaving the sagittal stratum more laterally, IFOF courses from the medial to the insula and external capsule in the deep white matter of the temporal lobe (34). There is no consensus about the MdLF functions. MdLF is thought to have a role in language and attention functions because it has many connections with well-known cortical areas, including AG and STG $(31,32)$. Due to these connections, MdLF plays a role in translating sounds to articulate form, processing words to acoustic and phonetic, and forming word (21). IFOF plays a key role in the semantic phase through processing of verbal stimulation as well as in the visual factors of communication; it is thought to play a role in the planning of motor functions, reading, and writing $(1,36)$. Our study with the case description also revealed the long association bundle connectivity and the importance of the knowing the courses of fibers, as we mentioned in the results of the dissection and FST. The case mostly related with these fibers and especially, we dissected the tumor from IC and AF created a cleavage between the tumor and the fibers. The FST is important in this stage to preserve the normal brain tissue covering the glioblastoma of the TPOJ. Correlation of the dissection and the surgical pictures as well as DTI and MRI images have a great potential to demonstrate several fiber pathways and their relationship in a stepwise manner in the preoperative stage. Understanding the fiber tracts trajectories and their relationships with each fiber after detailed 3D dissection can provide better surgical outcomes and less brain damage.

\section{CONCLUSION}

Surgical images supporting microsurgical fiber tract dissections are the best methods to learn the three-dimensional internal anatomy of the brain to improve the surgical competence. Understanding the courses and relationships of the fibers around the pathological lesion provides better surgical 
maneuverability. Using new and accurate techniques, including the FST, can minimize the risk for intraoperative brain injury. Excellent anatomical knowledge of the TPOJ and associated tracts is critical and plays a key role in managing intrinsic brain lesions, including gliomas and vascular malformations, and preventing surgical complications and iatrogenic brain damage.

\section{ACKNOWLEDGEMENT}

Preparation for publication of this article is partly supported by Turkish Neurosurgical Society.

\section{REFERENCES}

1. Almairac F, Herbet G, Moritz-Gasser S, de Champfleur NM, Duffau $\mathrm{H}$ : The left inferior fronto-occipital fasciculus subserves language semantics: A multilevel lesion study. Brain Struct Funct 220:1983-1995, 2015

2. Amaral DG, Price JL: Amygdalo-cortical projections in the monkey (Macaca fascicularis). J Comp Neurol 230:465-496, 1984

3. Bauman MD, Lavenex P, Mason WA, Capitanio JP, Amaral DG: The development of social behavior following neonatal amygdala lesions in rhesus monkeys. J Cogn Neurosci 16:1388-1411, 2004

4. Behin A, Hoang-Xuan K, Carpentier AF, Delattre JY: Primary brain tumours in adults. Lancet 361:323-331, 2003

5. Brauer J, Anwander A, Friederici AD: Neuroanatomical prerequisites for language functions in the maturing brain. Cereb Cortex 21:459-466, 2011

6. Burks JD, Boettcher LB, Conner AK, Glenn CA, Bonney PA, Baker CM, Briggs RG, Pittman NA, O'Donoghue DL, Wu DH, Sughrue ME: White matter connections of the inferior parietal lobule: A study of surgical anatomy. Brain Behav 7:e00640, 2017

7. Catani $M$, Jones $D K$, ffytche $D H$ : Perisylvian language networks of the human brain. Ann Neurol 57:8-16, 2005

8. Catani M, Mesulam M: The arcuate fasciculus and the disconnection theme in language and aphasia: History and current state. Cortex 44:953-961, 2008

9. Catani $\mathrm{M}$, Thiebaut de Schotten M: A diffusion tensor imaging tractography atlas for virtual in vivo dissections. Cortex 44:1105-1132, 2008

10. Chechlacz M, Rotshtein P, Hansen PC, Deb S, Riddoch MJ, Humphreys GW: The central role of the temporo-parietal junction and the superior longitudinal fasciculus in supporting multi-item competition: Evidence from lesion-symptom mapping of extinction. Cortex 49:487-506, 2013

11. De Benedictis A, Duffau H, Paradiso B, Grandi E, Balbi S, Granieri E, Colarusso E, Chioffi F, Marras CE, Sarubbo S: Anatomo-functional study of the temporo-parieto-occipital region: Dissection, tractographic and brain mapping evidence from a neurosurgical perspective. J Anat 225:132-151, 2014

12. Duffau H, Gatignol P, Mandonnet E, Peruzzi P, TzourioMazoyer N, Capelle L: New insights into the anatomofunctional connectivity of the semantic system: A study using cortico-subcortical electrostimulations. Brain 128:797-810, 2005
13. Duffau H, Thiebaut de Schotten M, Mandonnet E: White matter functional connectivity as an additional landmark for dominant temporal lobectomy. J Neurol Neurosurg Psychiatry 79:492-495, 2008

14. Fernandez-Miranda JC, Rhoton AL Jr, Alvarez-Linera J, Kakizawa Y, Choi C, de Oliveira EP: Three-dimensional microsurgical and tractographic anatomy of the white matter of the human brain. Neurosurgery 62:989-1026; discussion 1026-1028, 2008

15. Fernandez-Miranda JC, Wang Y, Pathak S, Stefaneau L, Verstynen T, Yeh FC: Asymmetry, connectivity, and segmentation of the arcuate fascicle in the human brain. Brain Struct Funct 220:1665-1680, 2015

16. Friederici AD: Language development and the ontogeny of the dorsal pathway. Front Evol Neurosci 4:3, 2012

17. Geesaman BJ, Born RT, Andersen RA, Tootell RB: Maps of complex motion selectivity in the superior temporal cortex of the alert macaque monkey: A double-label 2-deoxyglucose study. Cereb Cortex 7:749-757, 1997

18. Gerritsen JKW, Vietor CL, Rizopoulos D, Schouten JW, Klimek M, Dirven CMF, Vincent AJE: Awake craniotomy versus craniotomy under general anesthesia without surgery adjuncts for supratentorial glioblastoma in eloquent areas: A retrospective matched case-control study. Acta Neurochir (Wien)2019

19. Gierhan SM: Connections for auditory language in the human brain. Brain Lang 127:205-221, 2013

20. Gravesteijn BY, Keizer ME, Vincent A, Schouten JW, Stolker RJ, Klimek M: Awake craniotomy versus craniotomy under general anesthesia for the surgical treatment of insular glioma: Choices and outcomes. Neurol Res 40:87-96, 2018

21. Hickok G, Poeppel D: The cortical organization of speech processing. Nat Rev Neurosci 8:393-402, 2007

22. Hofman MA: Evolution of the human brain: When bigger is better. Front Neuroanat 8:15, 2014

23. Jennings JE, Kassam AB, Fukui MB, Monroy-Sosa A, Chakravarthi S, Kojis N, Rovin RA: The surgical white matter chassis: A practical 3-dimensional atlas for planning subcortical surgical trajectories. Oper Neurosurg (Hagerstown) 14:469-482, 2018

24. Kamali A, Flanders AE, Brody J, Hunter JV, Hasan KM: Tracing superior longitudinal fasciculus connectivity in the human brain using high resolution diffusion tensor tractography. Brain Struct Funct 219:269-281, 2014

25. Karas CS, Chiocca EA: Neurosurgical robotics: A review of brain and spine applications. J Robot Surg 1:39-43, 2007

26. Khashper A, Chankowsky J, Del Carpio-O’Donovan R: Magnetic resonance imaging of the temporal lobe: Normal anatomy and diseases. Can Assoc Radiol J 65:148-157, 2014

27. Klingler J: Erleichterung der makroskopischen Praeparation des Gehirns durch den Gefrierprozess. Schweiz Arch Neurol Psychiatr 36:247-256, 1935

28. Logothetis NK, Schall JD: Neuronal correlates of subjective visual perception. Science 245:761-763, 1989

29. Loui P, Alsop D, Schlaug G: Tone deafness: A new disconnection syndrome? J Neurosci 29:10215-10220, 2009 
30. Makris N, Kennedy DN, Mclnerney S, Sorensen AG, Wang R, Caviness VS Jr, Pandya DN: Segmentation of subcomponents within the superior longitudinal fascicle in humans: A quantitative, in vivo, DT-MRI study. Cereb Cortex 15:854-869, 2005

31. Makris N, Pandya DN: The extreme capsule in humans and rethinking of the language circuitry. Brain Struct Funct 213:343-358, 2009

32. Makris N, Preti MG, Asami T, Pelavin P, Campbell B, Papadimitriou GM, Kaiser J, Baselli G, Westin CF, Shenton ME, Kubicki M: Human middle longitudinal fascicle: Variations in patterns of anatomical connections. Brain Struct Funct 218:951-968, 2013

33. Makris N, Zhu A, Papadimitriou GM, Mouradian P, Ng I, Scaccianoce E, Baselli G, Baglio F, Shenton ME, Rathi Y, Dickerson B, Yeterian E, Kubicki M: Mapping temporoparietal and temporo-occipital cortico-cortical connections of the human middle longitudinal fascicle in subject-specific, probabilistic, and stereotaxic Talairach spaces. Brain Imaging Behav 11:1258-1277, 2017

34. Maldonado IL, de Champfleur NM, Velut S, Destrieux C, Zemmoura I, Duffau H: Evidence of a middle longitudinal fasciculus in the human brain from fiber dissection. J Anat 223:38-45, 2013

35. Malkova L, Mishkin M: One-trial memory for object-place associations after separate lesions of hippocampus and posterior parahippocampal region in the monkey. J Neurosci 23:1956-1965, 2003

36. Martino J, Brogna C, Robles SG, Vergani F, Duffau H: Anatomic dissection of the inferior fronto-occipital fasciculus revisited in the lights of brain stimulation data. Cortex 46:691-699, 2010

37. Martino J, da Silva-Freitas $\mathrm{R}$, Caballero $\mathrm{H}$, Marco de Lucas E, Garcia-Porrero JA, Vazquez-Barquero A: Fiber dissection and diffusion tensor imaging tractography study of the temporoparietal fiber intersection area. Neurosurgery 72:8797; discussion 97-98, 2013

38. Murray EA, Bussey TJ, Hampton RR, Saksida LM: The parahippocampal region and object identification. Ann N Y Acad Sci 911:166-174, 2000

39. O’Donnell LJ, Suter Y, Rigolo L, Kahali P, Zhang F, Norton I, Albi A, Olubiyi O, Meola A, Essayed WI, Unadkat P, Ciris PA, Wells WM 3rd, Rathi Y, Westin CF, Golby AJ: Automated white matter fiber tract identification in patients with brain tumors. Neuroimage Clin 13:138-153, 2017

40. Parlatini V, Radua J, Dell'Acqua F, Leslie A, Simmons A, Murphy DG, Catani M, Thiebaut de Schotten M: Functional segregation and integration within fronto-parietal networks. Neuroimage 146:367-375, 2017

41. Petrella JR, Shah LM, Harris KM, Friedman AH, George TM, Sampson JH, Pekala JS, Voyvodic JT: Preoperative functional MR imaging localization of language and motor areas: effect on therapeutic decision making in patients with potentially resectable brain tumors. Radiology 240:793-802, 2006
42. Petrides $M$, Pandya DN: Distinct parietal and temporal pathways to the homologues of Broca's area in the monkey. PLoS Biol 7:e1000170, 2009

43. Petrides M, Pandya DN: Projections to the frontal cortex from the posterior parietal region in the rhesus monkey. J Comp Neurol 228:105-116, 1984

44. Sarubbo S, Le Bars E, Moritz-Gasser S, Duffau H: Complete recovery after surgical resection of left Wernicke's area in awake patient: A brain stimulation and functional MRI study. Neurosurg Rev 35:287-292; discussion: 292, 2012

45. Schmahmann JD, Pandya DN, Wang R, Dai G, D'Arceuil HE, de Crespigny AJ, Wedeen VJ: Association fibre pathways of the brain: parallel observations from diffusion spectrum imaging and autoradiography. Brain 130:630-653, 2007

46. Smith JS, Chang EF, Lamborn KR, Chang SM, Prados MD, Cha S, Tihan T, Vandenberg S, McDermott MW, Berger MS: Role of extent of resection in the long-term outcome of lowgrade hemispheric gliomas. J Clin Oncol 26:1338-1345, 2008

47. Steinmetz MA, Motter BC, Duffy CJ, Mountcastle VB: Functional properties of parietal visual neurons: Radial organization of directionalities within the visual field. $J$ Neurosci 7:177-191, 1987

48. Stummer W, Reulen HJ, Meinel T, Pichlmeier U, Schumacher W, Tonn JC, Rohde V, Oppel F, Turowski B, Woiciechowsky C, Franz K, Pietsch T, Group AL-GS: Extent of resection and survival in glioblastoma multiforme: Identification of and adjustment for bias. Neurosurgery 62:564-576; discussion: 564-576, 2008

49. Thiebaut de Schotten M, Dell'Acqua F, Forkel SJ, Simmons A, Vergani F, Murphy DG, Catani M: A lateralized brain network for visuospatial attention. Nat Neurosci 14:1245-1246, 2011

50. Thiebaut de Schotten M, Urbanski M, Duffau H, Volle E, Levy $\mathrm{R}$, Dubois $\mathrm{B}$, Bartolomeo P: Direct evidence for a parietalfrontal pathway subserving spatial awareness in humans. Science 309:2226-2228, 2005

51. Vigneau M, Beaucousin V, Herve PY, Duffau H, Crivello F, Houde O, Mazoyer B, Tzourio-Mazoyer N: Meta-analyzing left hemisphere language areas: Phonology, semantics, and sentence processing. Neuroimage 30:1414-1432, 2006

52. Wu JS, Zhou LF, Tang WJ, Mao Y, Hu J, Song YY, Hong $\mathrm{XN}$, Du GH: Clinical evaluation and follow-up outcome of diffusion tensor imaging-based functional neuronavigation: A prospective, controlled study in patients with gliomas involving pyramidal tracts. Neurosurgery 61:935-948; discussion: 948939, 2007

53. Wu Y, Sun D, Wang Y, Wang Y, Wang Y: Tracing short connections of the temporo-parieto-occipital region in the human brain using diffusion spectrum imaging and fiber dissection. Brain Res 1646:152-159, 2016 